\title{
Testing Prerequisite Knowledge of Thermodynamics during a Thermody- namics II Course
}

\section{Dr. Kamau Wright, University of Hartford}

Kamau Wright is an assistant professor of mechanical engineering at the University of Hartford. He specializes in thermo-fluids and plasma engineering. His technical research interests include applications of high voltage plasma discharges to liquids and wastewaters; plasma decomposition of carbon dioxide; fouling prevention and mitigation for heat exchangers; oxidation of organic matter in water; and inactivation of bacteria using high voltage plasmas.

\section{Dr. Ivana Milanovic, University of Hartford}

Ivana Milanovic is a professor of mechanical engineering at the University of Hartford. Her area of expertise is thermo-fluids with research interests in vortical flows, computational fluid dynamics, multiphysics modeling, and collaborative learning strategies. Dr. Milanovic is a contributing author for more than 90 journal articles, NASA reports, conference papers, and software releases.

\section{Dr. C. Cy Yavuzturk, University of Hartford}




\title{
Testing prerequisite knowledge of thermodynamics during a Thermodynamics II course
}

\begin{abstract}
A surprise 40-min Pre-requisite Exam was administered in the first week of a Thermodynamics II course to assess pre-requisite knowledge of Thermodynamics I curriculum, and achievement of associated learning outcomes. This exam placed particular interest on students' understanding of concepts related to transient analysis. Students' performance on this Pre-requisite Exam would not negatively impact their grades in Thermodynamics II, and if at all counted, could be used toward extra credit. The Pre-requisite Exam was mostly motivated by reported/perceived positive enhancements in sections of Thermodynamics I, and interest in identifying and demonstrating additional robust yet pragmatic methods of assessing the extent to these enhancements. From the instructors' perspectives, correlations might potentially be able to be made between Pre-requisite Exam performance and previous Thermodynamics I experiences, addressing questions such as, "what might be the effect of the time between a student successfully completing the pre-requisite course, and performance in the post-requisite Thermodynamics II course?"; "can the impact of any particular student experiences or teaching and learning strategies implemented in the pre-requisite course be identified as positively affecting performance and achievement in the post-requisite Thermodynamics II course?" The Pre-requisite Exam administered in the beginning of a Thermodynamics II course provides a data point in addressing such questions. The primary motivation of this study was to provide further assessment of positive results that were obtained in implementing collaborative projects with simulation components as high impact practices into a Thermodynamics I course. The results of the present study have indicated that the implementation of such a collaborative project into sections of Thermodynamics I did not directly result in better performance on the specific prerequisite exam problem. Further, it was observed that the amount of time that had passed since a student had completed the Thermodynamics I course, did not impact performance on this exam problem. The method of administering Pre-requisite Exams is demonstrated and suggested as a method which can be strategically utilized to uncover, describe, and assess the impact (or lack thereof) of specific perceived enhancements in the curriculum.
\end{abstract}

\section{Introduction}

With piqued interest in the implementation of new and/or improved strategies and innovations in the delivery of engineering material, educators must find equally strategic yet pragmatic methods to assess these perceived innovations. Sure, the graded assignments, quizzes, projects, exams, and more, that are administered can supply such data and information, and are designed to do as much. Still, additional data points can be useful, as there can be nuances and situation-specific experiences that might result in variations in student performance, from month to month, or semester to semester. Further, when viewed by engineering educators through the lens of programmatic goals and performance of wider cohorts of students, true innovations must be tested across sections and time, to establish the actual robustness (or lack thereof) of such strategies, or at least provide benchmarks for improvement.

It is in this spirit that a "pop-exam," or surprise exam was administered in the first week of a Thermodynamics II course to assess pre-requisite knowledge of Thermodynamics I curriculum, and achievement of associated learning outcomes. For this specific exam, one aspect 
of students' performance which was on display was their understanding of thermodynamics concepts related to transient analysis. While their performance on this Pre-requisite Exam did not count toward their Thermodynamics II grade, it could be used to help jolt students back into the mode of thermodynamics, encouraging them to study and or be aware of any gaps that they should fill on the way to being successful in the Thermodynamics II course.

Instructors could also utilize these extra data points, in a similar way, scaffolding extra support for certain concepts as necessary, comparing students' performance in regard to related areas, on future Thermodynamics II quizzes or exams. Further, in regard to the thermo-fluids courses in mechanical engineering and programmatic goals, when viewed in light of students' academic backgrounds, questions could be addressed such as, "what might be the impact of the time between a student successfully completing a pre-requisite course, and performance in the post-requisite Thermodynamics II course," or "can the impact of any particular student experiences or teaching and learning strategies implemented in the pre-requisite course be identified as positively impacting performance and achievement in the post-requisite Thermodynamics II course?"

One specific motivation for this work was to conduct follow-up analysis to try and further observe the impact or lack thereof of implementing select practices into a Thermodynamics I course. While previous studies have demonstrated the benefits of a collaborative project (CP) with a simulation component on students' understanding, interest, and overall performance, it was interesting to see if any of these effects could be observed over time, through a Pre-requisite Exam in a post-requisite course. Capturing the true essence, effect, and potential of new teaching enhancements in real-time can be a challenge. Of course there are many factors, some named and most not, which can affect this kind of study or alter the results. Still, with that being well known, this work is developed in an attempt to help push forward potential ways to establish clear, tangible, and effective approaches for determining positive enhancements in the delivery of curriculum in the classroom. For example, if there is to be developed, a method which demonstrates much more than incremental improvements to teaching and learning, and supersedes all instructor-, environment-, and student-related variables, then an accompanying effective assessment method utilizing a Pre-requisite Exam with carefully selected problem(s) might be an effective way to demonstrate such enhancements.

The main goal here is to establish assessment practices which impact the overall program, and the students. The second goal would then be identification of most positive or valuable experiences, if any, during students' Thermodynamics I and Thermodynamics II semesters. Looking at previous Thermodynamics I experiences, could help stimulate enhancement in future Thermodynamics I sections. Observing present Thermodynamics II activities, might lead to identification of activities, if any, which can help improve any of students' knowledge gaps and associated performance, whether they love thermo-fluids, are typically great students, or are on the opposite ends of this spectrum. While it could be expected that consistent trends might not be uncovered, or any perceived trends that were uncovered still might not be ultimately indicative of the factors that lead to the Prerequisite Exam outcomes, this approach does help in providing data points that may outline potential promising areas for future investigation. These efforts are all toward the goals of improving teaching strategies; programmatic strengths; achievement of learning outcomes; and helping students to retain prerequisite knowledge during their academic careers and beyond. It is expected that these are issues of interest to most if not all engineering 
programs as considerations are made on the impact of educational activities on students in the short, mid, and long- term.

\section{Interest in pre-requisite knowledge in engineering}

While grades remain the primary overall indicator of student learning in a course, the consideration of students' retaining of knowledge for deployment in post-requisite courses, and subsequent experiences, is of interest in engineering education and related fields [1-8]. These efforts can provide insight not only on achievement of specific course outcomes by individual students, but by cohorts of students. Further, strategies for strengthening or reactivating such prerequisite knowledge can be developed. Laman and Brannon [1] described a need to integrate prerequisite materials in a structural design of foundations course, utilizing electronic media such as pencasts to archive related supplemental resources, for potential delivery through an online tool. Dunsworth and $\mathrm{Wu}$ [2] discussed review of prerequisites in a senior level biomechanics course, by using a flipped model in which videos of select technical topics from prerequisite courses were made available to students and coupled with online quizzes, and required across the semester prior to topics where the technical knowledge would be needed.

The use of a test to assess students' knowledge before instruction (and after) includes Concept Inventory (CI) tests, historically requiring a forced choice between correct concepts and common-sense alternatives, and used in physics education [3]. Lanning et. al. [4] evaluated students' depth of prerequisite knowledge in a laboratory-based Aerospace Engineering Failure course, using a concept inventory exam. Evans et. al. [5] described progress on development of Concept Inventory assessment instruments, which could be used by instructors to judge the adequacy of their instruction, and upon inclusion of data segregated by how content was managed and delivered, to identify effective practices, and relate specific teaching techniques to student learning across multiple subjects. These subjects included thermodynamics; solid mechanics; signals and processing; electromagnetics; fluid mechanics; heat transfer, and more [5]. In discussing development of Concept Inventory assessment instruments related to the subject thermal and transport phenomena (e.g. thermodynamics, fluid mechanics, heat transfer), key concepts were identified of high importance and low student understanding to use as the focus for creating concept inventory, including heat vs. energy, heat vs. temperature, internal energy vs. enthalpy, steady-state vs. equilibrium processes, system vs. control volume analysis, amongst others [5]. The Prerequisite Exam administered as part of the present study presented students with a transient tank filling type problem which challenge them to discern between system vs. control volume analysis, and consider the concepts of steady state (or lack thereof) within their solution.

In regard to studies specific to thermodynamics, Bluestein tested undergraduate students in mechanical engineering technology for prerequisite knowledge in thermodynamics as an assessment tool [6]. Thompson et. al. [7] used a pretest in a Physical Thermodynamics course about two-thirds of the way through the course, to explore student understanding of mathematical concepts when applied in thermal physics contexts, specifically partial differentiation, before an introductory lesson on the Maxwell relations, which equate mixed second partial derivatives of various state functions. Karimi and Manteufel [8] examined the relationship between student learning in Thermodynamics I as indicated by grades, and subsequent performance in Thermodynamics II. 
The motivations and reasons for the different methods to address pre-requisite knowledge in a post-requisite course can be varied.

Data presented by Karimi and Manteufel [8] on grades in Thermodynamics I at their institution, offered 55 times and taught by nine instructors between fall semester 1994 and fall 2012, indicated that there were small variations in student passing rates for a given instructor from semester to semester. Although this could be caused by various factors, they explored the idea that a grade received in a single course might not necessarily signify the level of student knowledge and competency in the topics covered in that course, and they decided to track students' performance in the post-requisite Thermodynamics II course. The study concluded that while grades remain the primary overall indicator of student learning (where a high grade indicated superior mastery of the material and a failing grade indicates otherwise) grades are highly dependent on the instructor teaching the course, with data appearing to indicate that nontenure track faculty at their institution issue higher grades, and students passing Thermodynamics I with non-tenure track faculty are less prepared for Thermodynamics II.

Bluestein [6] upon finding it necessary over ten years to review knowledge of the prerequisite courses of calculus and Thermodynamics I extensively, at the beginning of a Thermodynamics II course, implemented a brief, in-class, closed book test on the first day of class in Thermodynamics II. This test is described as consisting of multiple choice questions on simple differentiation and integration (six questions) plus thermodynamics (four questions), and is mentioned to have been completed anonymously to relieve anxiety and to insure a true measure of what the students know as a group, with a three semester average under 50\% [6]. Conversely, it can be argued that if the test were not anonymous and additionally were assigned some sort of credit, this could also help it to be a true measure of students' performance. Bluestein [6] concluded that students in an advanced thermodynamics course were not sufficiently competent in the prerequisites for this course, and that their program intended to continue such pre-requisite testing in Thermodynamics II. Further, they wanted to explore the reason for the problem of retention of prerequisite material, including among potential reasons that the time factor (how long since students had completed the prerequisite thermodynamics course) might play a role in the results.

The goal of the present study was to test out an assessment approach, in hopes of retrospectively uncovering additional benefits of curricular innovation in a previous course (Thermodynamics I), as compared to other recent sections of the previous course. This study only describes performance on a Pre-requisite Exam in consideration of a univariate analysis of impact of elapsed time since completion of Thermodynamics I, and a univariate analysis of whether students were previously involved in a Collaborative Project (CP) or not. Other factors were not considered here. Subsequent studies may also include the mining of data to provide a control for students' performance in Thermodynamics I, with the expectation that a student achieving a grade of $\mathrm{C}$ - would not have mastered (and retained) material at the same level as a student who achieved an A even if the same amount of time has elapsed. Additional data that can be uncovered might include the number of times that Thermodynamics I was taken (and repeated) before obtaining a grade of $\mathrm{C}$-. 


\section{Thermodynamics I and the Pre-requisite Exam in Thermodynamics II}

Thermodynamics I (ME 236) is a mandatory 3-credit course in mechanical engineering, offered at the authors' University, and similarly for mechanical engineering programs across the globe. Prerequisite(s) for this course at this institution, include Calculus II and Calculus-based physics II. Select topics, as described in the mechanical engineering departments' course description include:

- Theoretical and applied classical engineering thermodynamics of non-reacting substances;

- The first and second laws

- The properties of ideal and real substances

- Gas mixtures

- The behavior of closed and open systems for reversible and irreversible processes

- Thermodynamic cycles

Learning outcomes can be generated which reflect, and build on these topics. Sample learning outcomes include but are not limited to:

- Know the units, symbols and vocabulary of thermodynamics

- Use traditional thermodynamic tables and diagrams as well as software-based tables to successfully solve thermodynamic problems

- Know how to apply mass and energy balances to a variety of simple processes

- Communicate thermodynamic problems and their solutions with words, schematic diagrams and mathematical expressions

- Interpret thermodynamic terminology

- Distinguish between closed systems and open control volumes, and demonstrate understanding of the behavior of such systems for reversible and irreversible processes

- Create schematics that include labels, which are useful to thermodynamic analysis

- Evaluate, locate, and write appropriate thermodynamic property data and values for ideal and real substances

- Demonstrate understanding of and ability to apply the conservation of mass, and first and second laws of thermodynamics, in consideration of steady state and transient analysis

Typically the first half-a-dozen chapters in widely used thermodynamics textbooks in mechanical engineering cover the following topics or some combination of these, many times in this order although variations in the text or the delivery of these topics can be observed:

(1) Introductory concepts

(2) Properties of a pure substance

(3) The first law of thermodynamics

(4) Energy analysis for a control volume

(5) The second law of thermodynamics

(6) Entropy 
Examples of texts which utilize this general format include the Fundamentals of Thermodynamics text by Borgnakke and Sonntag [9], Thermodynamics: An Engineering Approach by Cengel and Boles [10], and the Fundamentals of Engineering Thermodynamics text by Moran, Shapiro, Boettner, and Bailey [11]. It should be noted that in addition to the chapter topics mentioned which must be covered in a mechanical engineering Thermodynamics I course, there can be room toward the end of a course, for introduction of various types of more complex versions of systems described in earlier chapters, including vapor power systems (i.e., with phase change), gas power systems (i.e., with gaseous working fluids), refrigeration and heat pump systems (with phase change or with gaseous working fluids). Addressing these such topics can help build a bridge to subsequent thermodynamics-related courses, or introduce these topics for programs in which there is not a Thermodynamics II or Applied Thermodynamics course, or such a course is optional.

In select sections of the Thermodynamics I courses in recent years at the author's institution, a new approach to facilitating learning of an engineering thermodynamics course, was implemented, which included the use of themed collaborative projects with a simulation component, relying mostly on the use of COMSOL Multiphysics® software $[12,13]$. While benefits were reported including providing students with interactivity, enhancements in students' perceptions that they understood thermodynamics, and also better average performance on select final exam questions, toward the goal of continued assessment and improvement, the present study sought to establish additional data points for analysis of the engineering education innovation. Hence as part of the authors deciding to administer Pre-requisite Exams in Thermodynamics II, it was hypothesized that the collaborative project might have boosted students' capabilities to tackle thermodynamics problems which included transient analysis.

Hence, while a range of thermodynamics problems could have been selected for the Prerequisite Exam, which might help test a more foundational knowledge of the subject, based on the general concepts previously described, a problem was selected which would require students to consider these concepts in regard to transient analysis in thermodynamics. The specific problem chosen for assessment in the Prerequisite Exam included but was not limited to the following learning outcomes: (a) distinguish between closed systems and open control volumes; (b) apply the conservation of mass in consideration of transient analysis; and (c) apply the first law of thermodynamics to a control volumes $(\mathrm{CV})$ and in consideration of transient analysis. While there were various other learning outcomes which were a part of the Pre-requisite Exam, in general, understanding of "transient analysis" in thermodynamics was a primary focus for this assessment.

The format of the exam required students to solve one problem with multiple parts. This was not a concept question. It was a question involving filling or emptying of a vessel. The problem selected was not particularly aligned with the subject matter that would be studied in the Thermodynamics II course, with lots of focus on cycles, but the foundational knowledge required to address this problem was indeed necessary. This included but was not limited to, identification and consideration of the need to discern between the use of closed system analysis or control volume analysis; the use of property data, and the application of the $1^{\text {st }}$ Law of Thermodynamics. In subsequent instances of the utilization of this assessment method, it is anticipated that different types of problems will be selected for different reasons. The problem 
during this iteration had the main focus of looking at students abilities to consider thermodynamics through the lens of transient analysis.

It should be noted that previous results obtained on the effectiveness of the $\mathrm{CP}$, may have also benefited from connection between emphasized topics in course material covered and select exam questions, both in format of problems solved during the CP experience in Thermodynamics I, and requirements. This Pre-requisite Exam approach was an intended way to help eliminate some of those potential factors, while still indicating enhancements. It is suggested here that curricular innovations be held to this level of scrutiny, toward the goal of maximizing datadriven improvement.

A rubric was developed for grading the 40-minute thermodynamics Prerequisite Exam, and an associated method for grading and assigning points was established, strengthened by instructor expertise, and years of experience, and tempered with the consultation and insights of various resources, rubrics, and assessment schemes related to STEM exam problems such as physics courses [14-16], and TA grading in physics courses [17]. After the exams were graded, the scores were each normalized against the overall average, to help categorize the distribution of student performance about the mean. There were 45 students who were administered the Prerequisite Exam in the first week of class. However, one of those students had not actually satisfied the Thermodynamics I required grade of $\mathrm{C}^{-}$, and was dropped from the class in that week. Hence results are presented for a total of $n=44$ students across multiple sections of Thermodynamics II (ME 337). Information about students' background Thermodynamics I experiences were mined, including how long ago the course had been completed, and whether or not the student had a collaborative project in Thermodynamics I. This data was combined with normalized performance data on the Pre-requisite Exam, and then all of this data was deidentified, and prepared to be presented in aggregate. It should be noted that, although the Prerequisite Exam grade did not count toward students' standard Thermodynamics II grade, it could be used to help jolt students back into the mode of thermodynamics, encouraging them to go back and study and or be aware of any gaps that they should fill on the way to being successful in the Thermodynamics II course. Other than for general assessment of students' abilities, as they entered the Thermodynamics II course, students were not made aware of any other specific motivations of the exam. However, performance could be considered for extra credit at the instructor's discretion.

\section{Impact of Thermodynamics I experience: collaborative projects and time since completion}

The impact or lack thereof of timing (when and how long ago, Thermodynamics 1 was completed for a grade $\geq \mathrm{C}^{-}$?) can be observed. With the Thermodynamics II course offered in the fall, and the Thermodynamics I course offered in the spring, there is typically at least a fewmonths gap between when students complete these courses. With data also available for students who completed Thermodynamics I in the summer before Thermodynamics II, the consequence, if any, of being away from the material during the "summer semester" could be analyzed given the data set. Further, the overall effect of the amount of time since completing Thermodynamics I could be considered. Table 1 shows a comparison of performance for groups of students who completed Thermodynamics 1 with a $\mathrm{C}$ - or above at some time within the past few semesters. For these purposes, completing Thermodynamics I during a summer semester is counted as completing it "one semester ago." Completing Thermodynamics I in the previous spring is 
counted as "two semesters ago", and so on. Data for students who transferred in Thermodynamics I credit from a different university are grouped separately. For each of these groups, the average normalized scores, standard deviations of these normalized scores, maximum single score, and minimum single score, are shown in Table 1.

Table 1: Normalized performance on Pre-requisite Exam

\begin{tabular}{l|llll}
\hline $\begin{array}{l}\text { When/how student completed } \\
\text { Thermodynamics I? }\end{array}$ & Average & Standard Deviation & Maximum & Minimum \\
\hline One semester ago $(\mathrm{n}=7)$ & 1.07 & \pm 0.58 & 1.57 & 0.24 \\
Two semesters ago $(\mathrm{n}=20)$ & 1.01 & \pm 0.84 & 2.54 & 0.00 \\
Three semesters ago $(\mathrm{n}=0)$ & -- & -- & -- & -- \\
Four semesters ago $(\mathrm{n}=1)$ & 1.21 & -- & 1.21 & 1.21 \\
Five semesters ago $(\mathrm{n}=2)$ & 1.15 & \pm 0.43 & 1.45 & 0.85 \\
Transferred in credit $(\mathrm{n}=14)$ & 0.98 & \pm 0.79 & 2.42 & 0.12 \\
\hline All students $(\mathrm{n}=44)$ & 1.00 & \pm 0.75 & 2.54 & 0.00 \\
\hline
\end{tabular}

The impact or lack thereof of transferring in a credit for Thermodynamics 1, i.e., completing it at a different school, as opposed to completing it at this university can also be observed in Table 1.

With the motivation of attempting to further observe impacts or the lack thereof of implementation of a select $\mathrm{CP}$ as a high impact practice, into sections of a Thermodynamics I course, the data was also split up by CP Students vs. Non-CP students.

The average scores between CP students and non-CP students were negligible. Students who were exposed to a collaborative project in their Thermodynamics I course, had scores which were slightly below the average normalized score of 1 , while students who were not exposed to a $\mathrm{CP}$ in Thermodynamics 1 had scores slightly above the average. These values are shown in the Table. It is safe to say that with the scores so close to average and the standard deviations so large, there was no significant difference in the scores between $\mathrm{CP}$ and non-CP students.

In considering the maximum and minimum normalized scores on the Pre-requisite Exam, the maximum performance was by a CP student scoring $2.54 \mathrm{x}$ the average of all students. The minimum performance was a non-CP student scoring $0 \mathrm{x}$ the average of all students. The average, standard deviation, minimum, and, maximum scores for $\mathrm{CP}$ and non- $\mathrm{CP}$ students are indicated in the table.

Table 2: Normalized performance on Pre-requisite Exam: CP students vs. Non-CP students

\begin{tabular}{l|llll}
\hline & Average & Standard Deviation & Maximum & Minimum \\
\hline CP Students $(\mathrm{n}=9):$ & 0.97 & \pm 0.78 & 2.54 & 0.12 \\
Non-CP Students $(\mathrm{n}=35):$ & 1.01 & \pm 0.75 & 2.42 & 0.00 \\
\hline All students $(\mathrm{n}=44)$ & 1.00 & \pm 0.75 & 2.54 & 0.00 \\
\hline
\end{tabular}


As described and illustrated in Table 2, the differences between performances of CP students vs. non-CP students on the Pre-requisite Exam were negligible.

\section{Discussion}

Again, the main focus of the study was assessment of perceived benefits in sections of a Thermodynamics I course. In subsequent iterations of this approach, different problems can be selected for a variety of reasons; a wider range of variables can be considered, and a strategic choice can be made of what to do in response to such results, in Thermodynamics II, in the way of offering credit and/or providing students with feedback or interventions based on their performance.

In regard to what an acceptable score might be on a Pre-requisite Exam, the present study makes no comments about this, choosing instead to attempt to observe if results of relative performance on a Pre-requisite Exam indicate great benefits in curricular innovations in select sections of a pre-requisite course. While an acceptable score might be based on the type of question asked, and the type of learning outcome being tested, the normalization of scores on specific topics, for identification of more than marginally better effectiveness in teaching approaches was the main focus. Further, it can be commented that the exam given was not an easy one at all for students, with one student who had transferred into the university that semester that the Prerequisite Exam was administered (i.e., had an exam during the first day at a new school), retrospectively (a semester later) describing the Prerequisite Exam in a jovial manner, but as impossible to solve (it was indeed possible, although not the easiest problem).

In addition to the potential impact within the class, this general method can inform the strengths and areas for improvement in the curriculum of engineering programs at institutions. Universal tests (i.e., standardized tests or finals) could potentially have a similar outcome, however, by framing this as Pre-requisite test in Post-requisite courses, this approach can serve as formative rather than summative assessment. Depending on how results are disseminated, this can help students improve their achievement, and instructors improve their programs through enhancements in the curriculum. It can also help instructors' efforts at developing and assessing curricular innovations.

This procedure of administering a Pre-requisite Exam is one which can play a different role than course grades for an engineering program, much like a standardized test can play in tandem with grades of an institution, except with the additional and direct benefit of assessing curricular innovations and programmatic goals, and offering students' a chance to fill gaps in knowledge, and/or improve their performance and overall achievement.

The consideration not only of students' previous performance in prerequisite courses, but in their ability to recall and re-apply this pre-requisite knowledge in subsequent experiences is important. And once this is assessed, there are options for what can be done in response. As described, results can be used to further enhance the overall engineering program, including the prerequisite courses themselves; results can also be used in real time to scaffold support for the post-requisite class. Both can be done, as subsequent iterations of the post-requisite course may be planned which already take into account potential gaps in knowledge of Thermodynamics I, regardless of the reason. This includes students with $\mathrm{C}$-level performance to A-level 
performance, whether there are knowledge gaps to fill, or there is benefit to be realized by quick reactivation of knowledge early on in the semester. This also benefits instructors who have certain topics that they'd like to emphasize or use to engage students in higher level thinking, but could benefit from first having students brought "back up to speed." For example, Dunsworth and Wu's [2] "flipped review" procedure provides a model which address this by preemptively having students complete technical review video-quiz combos to strategically strengthen prerequisite knowledge before it was needed during the semester of a post-requisite course. Further, they indicated that the flipped review approach to ensuring students had pre-requisite knowledge, not only saved class time, and allowed them to repurpose as much as 2-3 weeks of classes, usually reserved for face-to-face lecture time in reviewing fundamental ME concepts, but provided more time for students to learn at a higher cognitive level [2].

The primary motivation for better understanding students' prerequisite knowledge can be in assessing curricular innovation, as it is in the present study, but can also be stimulated by simply wanting to better understand needs in approaching a post-requisite course. Instructors have long described anecdotally and formally [1-8], the importance of prerequisite knowledge for a student to be successful in his or her chosen degree program, and the impact that a lack of prerequisite knowledge can also have on an entire class, as an instructor may have to use valuable time to re-teach concepts that should have been previously learned [4]. Lanning et. al. [4], in evaluation of students' depth of prerequisite knowledge in an Aerospace Engineering course, administered a concept inventory exam at three points: at the end of a prerequisite course; at the beginning a post requisite course; and at the end of a post-requisite course, a procedure which allowed not only for testing of this prerequisite knowledge, but could indicate students' improvement in understanding over time. They [4] were able to plan revisions to the lecture and laboratory based upon reflection on the students' scores, in addition to establishing data which indicated $10 \%$ improvement by students on the exam over the course of the semester.

One concern in the implementation of the Prerequisite Exam in the present study is that since the students were not given a hard guarantee that it would contribute to their grade (or by how much), even some small amount, they might not have given an honest effort. As such, the results of the assessment method might be less meaningful. In future iterations of this approach, points will be assigned to this task that are proportional to performance, preferably as agreed upon by instructors across sections. While this does not appear to be the case in the current situation, based on anecdotal feedback, it is offered as a consideration for future iterations of this type of approach. It is evident that such a procedure would at the least, help promote the meaningfulness of the results, and at best, help guarantee students a chance to obtain a few extra points toward their performance in a challenging course. The data could also be used to construct an approach for review classes to make sure the student are prepared to be successful, or used to see how changes in the first class can stimulate increased retention of knowledge and higher order thinking by students. Since the same instructors are mostly involved in teaching both Thermodynamics I (either with or without CP) and Thermodynamics II, and collaboration and coordination is possible, enhancements are anticipated.

\section{Conclusion}

Causes for the distribution of grades, and consequences of different student background experiences on performance on the pre-requisite exam is not clear, as there are too many factors 
to discern. The impact of the High Impact Practices implemented into prior sections of Thermodynamics I was not observed through this method of testing pre-requisite knowledge of Thermodynamics I curriculum. Still, this attempt represents a set of data points in a larger effort to assess the effectiveness of new approaches in undergraduate engineering education, at the authors' institution. Some interesting findings in the data include the fact that the amount of time since completing Thermodynamics 1 did not have a significant effect on the average performance on the administered Pre-requisite Exam question. Additionally, although enhancements have been observed with the implementation of collaborative projects into Thermodynamics I courses, further impact was not demonstrated on the particular Pre-requisite Exam administered as part of the present study. While a range of potential limitations of the current analysis have been described, this study has helped to identify and develop an opportunity for independent methods for verification of perceived impacts on instructional interventions, in thermo-fluids courses. Future work includes demonstration of continued enhancements in teaching and learning methods in the described thermo-fluid courses, and improvement of associated assessment plans, including strategic implementation of updated Prerequisite Exams, potentially facilitated by the use of online Learning Management Software (LMS) across courses for administering and even grading exams. Such methods can help provide objective data for a wide range of stakeholders including colleges, programs, and instructors of pre- and post-requisite courses. Increased strategic collaboration with prerequisite course instructors play a role in these methods, and as part of these efforts, correlation of student performance on developed Pre-requisite Exams, with later Thermodynamics II performance should also be established.

\section{References}

[1] J. A. Laman and M. L. Brannon, "Integration of prerequisite resource materials in a structural design of foundations course using pencasts," in 121st ASEE Annual Conference and Exposition: 360 Degrees of Engineering Education, 2014: American Society for Engineering Education.

[2] Q. Dunsworth and Y. E. Wu, "Effective Review of Pre-requisites: Using Videos to Flip the Reviewing Process in a Senior Technical Course," presented at the American Society for Engineering Education (ASEE) Annual Conference \& Exposition, Salt Lake City, UT, Jun $24-27,2018$.

[3] D. Hestenes, M. Wells, and G. Swackhamer, "Force concept inventory," The physics teacher, vol. 30, no. 3, pp. 141-158, 1992.

[4] D. B. Lanning, W. Lestari, and S. A. Waterhouse, "Evaluating Prerequisite Knowledge Using a Concept Inventory for an Engineering Failure Course," in American Society for Engineering Education, 2011: American Society for Engineering Education.

[5] D. Evans et al., "Progress on concept inventory assessment tools," in 33rd Annual Frontiers in Education, 2003. FIE 2003., 2003, vol. 1, pp. T4G-1: IEEE.

[6] M. Bluestein, "Testing for prerequisites in thermodynamics as an assessment tool," Proceedings of the 2001 American Society for Engineering Education Annual Conference \& Exposition vol. 6, p. 1, 2001.

[7] J. R. Thompson, B. R. Bucy, and D. B. Mountcastle, "Assessing student understanding of partial derivatives in thermodynamics," in AIP Conference Proceedings, 2006, vol. 818, no. 1, pp. 77-80: AIP. 
[8] A. Karimi and R. Manteufel, "Correlation of prerequisite course grades with student performance," in Proceedings from the ASEE Annual Conference and Exposition, Atlanta, GA, 2013.

[9] C. Borgnakke and R. E. Sonntag, Fundamentals of Thermodynamics, 9th ed. Hoboken, NJ: Wiley, 2017.

[10] Y. A. Cengel and M. A. Boles, Thermodynamics: an engineering approach. 2002.

[11] M. J. Moran, H. N. Shapiro, D. D. Boettner, and M. B. Bailey, Fundamentals of Engineering Thermodynamics, 8th ed. Wiley, 2014.

[12] K. Wright, I. Milanovic, and T. Eppes, "Implementing Collaborative Projects Using a National Academy of Engineering (NAE) Grand Challenge: Provide Access to Clean Water," presented at the ASEE 125th Annual Conference \& Exposition, Salt Lake City, Utah, USA, June 24 - 27, 2018.

[13] K. Wright, "Collaborative Projects with simulation assignments in mechanical engineering thermodynamics courses," International Journal of Mechanical Engineering Education, p. 0306419018803624, 2018.

[14] J. L. Docktor et al., "Assessing student written problem solutions: A problem-solving rubric with application to introductory physics," Physical review physics education research, vol. 12, no. 1, p. 010130, 2016.

[15] N. B. Hill, "MAUVE: a new strategy for solving and grading physics problems," The Physics Teacher, vol. 54, no. 5, pp. 291-294, 2016.

[16] W. K. Adams and C. E. Wieman, "Analyzing the many skills involved in solving complex physics problems," American Journal of Physics, vol. 83, no. 5, pp. 459-467, 2015.

[17] E. Marshman, R. Sayer, C. Henderson, and C. Singh, "Contrasting grading approaches in introductory physics and quantum mechanics: The case of graduate teaching assistants," Physical Review Physics Education Research, vol. 13, no. 1, p. 010120, 2017. 